\title{
Fast subexponential algorithm for non-local problems on graphs of bounded genus
}

\author{
Frederic Dorn ${ }^{1}$, Fedor V. Fomin ${ }^{1 \star}$, and Dimitrios M. Thilikos ${ }^{2 \star \star}$ \\ 1 Department of Informatics, University of Bergen, N-5020 Bergen, Norway, \\ frederic.dorn@ii.uib.no, fedor.fomin@ii.uib.no \\ 2 Departament de Llenguatges i Sistemes Informàtics, \\ Universitat Politècnica de Catalunya, Barcelona, Spain, \\ sedthilk@lsi.upc.edu
}

\begin{abstract}
We give a general technique for designing fast subexponential algorithms for several graph problems whose instances are restricted to graphs of bounded genus. We use it to obtain time $2^{O(\sqrt{n})}$ algorithms for a wide family of problems such as Hamiltonian CyCle, $\Sigma$-embedded Graph Travelling Salesman Problem, Longest Cycle, and Max LEAF TREE. For our results, we combine planarizing techniques with dynamic programming on special type branch decompositions. Our techniques can also be used to solve parameterized problems. Thus, for example, we show how to find a cycle of length $p$ (or to conclude that there is no such a cycle) on graphs of bounded genus in time $2^{O(\sqrt{p})} \cdot n^{O(1)}$.
\end{abstract}

\section{Introduction}

Many common computational problems are NP-hard and therefore do not seem to be solvable by efficient (polynomial time) algorithms. However, while NPhardness is a good evidence for the intractability of a problem, in many cases, there is a real need for exact solutions. Consequently, an interesting and emerging question is to develop techniques for designing fast exponential or, when possible, sub-exponential algorithms for hard problems (see [15]).

The algorithmic study of graphs that can be embedded on a surface of small genus, and planar graphs in particular, has a long history. The first powerful tool for the design of sub-exponential algorithms on such graphs was the celebrated Lipton-Tarjan planar separator theorem $[9,10]$ and its generalization on graphs of bounded genus [7]. According to these theorems, an $n$-vertex graph of fixed genus can be "separated" into two roughly equal parts by a separator of size $O(\sqrt{n})$. This approach permits the use of a "divide and conquer" technique that provides subexponential algorithms of running time $2^{O(\sqrt{n})}$ for a wide range of combinatorial problems.

A similar approach is based on graph decompositions [6]. Here instead of separators one uses decompositions of small width, and instead of "divide and

* Additional support by the Research Council of Norway.

** Supported by the Spanish CICYT project TIN-2004-07925 (GRAMMARS). 
conquer" techniques, dynamic programming (here we refer to tree or branch decompositions - see Section 2 for details). The main idea behind this approach is very simple: Suppose that for a problem $\mathcal{P}$ we are able to prove that for every $n$-vertex graph $G$ of branchwidth at most $\ell$, the problem $\mathcal{P}$ can be solved in time $2^{O(\ell(G))} \cdot n^{O(1)}$. Since the branchwidth of an $n$-vertex graph of a fixed genus is $O(\sqrt{n})$, we have that $\mathcal{P}$ is solvable on $G$ in time $2^{O(\sqrt{n})} \cdot n^{O(1)}$.

For some problems like Minimum Vertex Cover or Minimum DominatING SET, such an approach yields directly algorithms of running time $2^{O(\sqrt{n})}$. $n^{O(1)}$ on graphs of bounded genus. However, for some problems, like HAMILtonian Cycle, $\Sigma$-embedded Graph TSP, Max Leaf Tree, and Steiner TREE, branchwidth arguments do not provide us with time $2^{O(\sqrt{n})} \cdot n^{O(1)}$ algorithms. The reason is that all these problems are "non-local" and despite many attempts, no time $2^{o(\ell(G) \log \ell)} \cdot n^{O(1)}$ algorithm solving these problems on graphs of branchwidth at most $\ell$ is known.

Recently, it was observed by several authors that if a graph $G$ is not only of branchwidth at most $\ell$ but is also planar, then for a number of "non-local" problems the $\log \ell$ overhead can be removed [3,5], resulting in time $2^{O(\sqrt{n})} \cdot n^{O(1)}$ algorithms on planar graphs. Similar result can be obtained by making use of separators [1].

It is a common belief that almost every technique working on planar graphs can be extended on graphs embedded on a surface of bounded genus. However, this is not always a straightforward task. The main difficulty in generalizing planar graph techniques $[1,3,5]$ to graphs of bounded genus is that all these techniques are based on partitioning a graph embedded on a plane by a closed curve into smaller pieces. Deineko et al. use cyclic separators of triangulations [1], Demaine and Hajiaghayi use layers of $k$-outerplanar graphs [3], and Dorn et al. sphere cut decompositions [5]. But the essence of all these techniques is that, roughly speaking, the situation occurring in the "inner" part of the graph bounded by the closed curve can be represented in a compact way by Catalan structures. None of these tools works for graphs of bounded genusseparators are not cyclic anymore, nor are there sphere cut decompositions and $k$-outerplanarity in non-planar graphs.

In this paper we provide a method to design fast subexponential algorithms for graphs of bounded genus for a wide class of combinatorial problems. Our algorithms are "fast" in the sense that they avoid the $\log n$ overhead and also because the constants hidden in the big-Oh of the exponents are reasonable. The technique we use is based on reduction of the bounded genus instances of the problem to planar instances of a more general graph problem on planar graphs where Catalan structure arguments are still possible. Such a reduction employs several results from topological graph theory concerning graph structure and noncontractible cycles of non-planar embeddings.

Our techniques, combined with the excluded grid theorem for graphs of bounded genus and bidimensionality arguments [2] provide also faster parameterized algorithms. For example we introduce the first time $2^{O(\sqrt{p})} \cdot n^{O(1)}$ algorithm for parameterized $p$-CYCLE which asks, given a positive integer $p$ and a 
$n$-vertex graph $G$, whether $G$ has a cycle of length at least $p$. Similar results can be obtained for other parameterized versions of non-local problems.

This paper is organized as follows. Towards simplifying the presentation of our results we decided to demonstrate how our approach works for the HAMILTONIAN CYCLE problem. Later, at the end of Section 4, we will explain how it can be applied to other combinatorial problems. We start with some basic definitions in Section 2 and some results from topological graph theory. Section 3 is devoted to the solution of HAMiltonian CyCLE problem (which asks if a given graph $G$ has a cycle containing all its vertices) on torus-embedded graphs. These graphs already inherit all "nasty" properties of non-planar graphs and all difficulties arising on surfaces of higher genus appear for torus-embedded graphs. However, the case of torus-embedded graphs is still sufficiently simple to exemplify the minimization technique used to obtained reasonable constants in the exponent. In Section 4, we explain how the results on torus-embedded graphs can be extended for any graphs embedded in a surface of fixed genus. Also in this section we discuss briefly applications of our results to parameterized algorithms on graphs of bounded genus.

\section{Definitions and preliminary results}

In this section we will give a series of definitions and results that will be useful for the presentation of the algorithms in Sections 3 and 4.

Surface embeddible graphs. We use the notation $V(G)$ and $E(G)$, for the set of the vertices and edges of $G$. A surface $\Sigma$ is a compact 2-manifold without boundary (we always consider connected surfaces). We denote by $\mathbb{S}_{0}$ the sphere $\left(x, y, z \mid x^{2}+y^{2}+z^{2}=1\right)$ and by $\mathbb{S}_{1}$ the torus $\left(x, y, z \mid z^{2}=1 / 4-\left(\sqrt{x^{2}+y^{2}}-\right.\right.$ $\left.1)^{2}\right)$. A line in $\Sigma$ is subset homeomorphic to $[0,1]$. An $O$-arc is a subset of $\Sigma$ homeomorphic to a circle. Whenever we refer to a $\Sigma$-embedded graph $G$ we consider a 2-cell embedding of $G$ in $\Sigma$. To simplify notations we do not distinguish between a vertex of $G$ and the point of $\Sigma$ used in the drawing to represent the vertex or between an edge and the line representing it. We also consider $G$ as the union of the points corresponding to its vertices and edges. That way, a subgraph $H$ of $G$ can be seen as a graph $H$ where $H \subseteq G$. We call by region of $G$ any connected component of $(\Sigma \backslash E(G)) \backslash V(G)$. (Every region is an open set.) A subset of $\Sigma$ meeting the drawing only in vertices of $G$ is called $G$-normal. If an $O$-arc is $G$-normal then we call it noose. The length of a noose $N$ is the number of its vertices and we denote it by $|N|$. Representativity [12] is the measure how dense a graph is embedded on a surface. The representativity (or face-width) $\operatorname{rep}(G)$ of a graph $G$ embedded in surface $\Sigma \neq \mathbb{S}_{0}$ is the smallest length of a noncontractible noose in $\Sigma$. In other words, $\operatorname{rep}(G)$ is the smallest number $k$ such that $\Sigma$ contains a noncontractible (non null-homotopic in $\Sigma$ ) closed curve that intersects $G$ in $k$ points. Given a $\Sigma$-embedded graph $G$, its radial graph (also known as vertex-face graph) is defined as the the graph $R_{G}$ that has as vertex set the vertices and the faces of $G$ and where an edge exists iff it connects a face and a vertex incident to it in $G$ ( $R_{G}$ is also a $\sigma$-embedded graph). If the 
intersection of a noose with any region results into a connected subset, then we call such a noose tight. Notice that each tight noose $N$ in a $\Sigma$-embedded graph $G$, corresponds to some cycle $C$ of its radial graph $R_{G}$ (notice that the length of such a cycle is $2 \cdot|N|)$. Also any cycle $C$ of $R_{G}$ is a tight noose in $G$. As it was shown by Thomassen in [14] (see also Theorem 4.3.2 of [11]) a shortest noncontractible cycle in a graph embedded on a surface can be found in polynomial time. By Proposition 5.5.4 of [11]) a noncontractible noose of minimum size is always a tight noose, i.e. corresponds to a cycle of the radial graph. Thus we have the following proposition.

Proposition 1. There exists a polynomial time algorithm that for a given $\Sigma$ embedded graph $G$, where $\Sigma \neq \mathbb{S}_{0}$, finds a noncontractible tight noose of minimum size.

The Euler genus of a surface $\Sigma$ is $\mathbf{e g}(\Sigma)=\min \{2 \mathbf{g}(\Sigma), \tilde{\mathbf{g}}(\Sigma)\}$ where $\mathbf{g}$ is the orientable genus and $\tilde{\mathbf{g}}$ the nonorientable genus. We need to define the graph obtained by cutting along a noncontractible tight noose $N$. We suppose that for any $v \in N \cap V(G)$, there exists an open disk $\Delta$ containing $v$ and such that for every edge $e$ adjacent to $v, e \cap \Delta$ is connected. We also assume that $\Delta \backslash N$ has two connected components $\Delta_{1}$ and $\Delta_{2}$. Thus we can define partition of $N(v)=N_{1}(v) \cup N_{2}(v)$, where $N_{1}(v)=\left\{u \in N(v):\{u, v\} \cap \Delta_{1} \neq \emptyset\right\}$ and $N_{2}(v)=\left\{u \in N(v):\{u, v\} \cap \Delta_{2} \neq \emptyset\right\}$. Now for each $v \in N \cap V(G)$ we duplicate $v$ : (a) remove $v$ and its incident edges (b) introduce two new vertices $v^{1}, v^{2}$ and (c) connect $v^{i}$ with the vertices in $N_{i}, i=1,2 . v^{1}$ and $v^{2}$ are vertices of the new $G$-normal $O$-arcs $N_{X}$ and $N_{Y}$ that border $\Delta_{1}$ and $\Delta_{2}$, respectively. We call $N_{X}$ and $N_{Y}$ cut-nooses. Note that cut-nooses are not necessarily tight (In other words, a cut-noose can enter and leave a region of $G$ several times.) The following lemma is very useful in proofs by induction on the genus. The first part of the lemma follows from Proposition 4.2.1 (corresponding to surface separating cycle) and the second part follows from Lemma 4.2.4 (corresponding to non-separating cycle) in [11].

Proposition 2. Let $G$ be a $\Sigma$-embedded graph where $\Sigma \neq \mathbb{S}_{0}$ and let $G^{\prime}$ be a graph obtained from $G$ by cutting along a noncontractible tight noose $N$ on $G$. One of the following holds

- $G^{\prime}$ can be embedded in a surface with Euler genus strictly smaller than $\mathbf{e g}(\Sigma)$. - $G^{\prime}$ is the disjoint union of graphs $G_{1}$ and $G_{2}$ that can be embedded in surfaces $\Sigma_{1}$ and $\Sigma_{2}$ such that $\mathbf{e g}(\Sigma)=\mathbf{e g}\left(\Sigma_{1}\right)+\mathbf{e g}\left(\Sigma_{2}\right)$ and $\mathbf{e g}\left(\Sigma_{i}\right)>0, i=1,2$.

Branchwidth. A branch decomposition of a graph $G$ is a pair $\langle T, \mu\rangle$, where $T$ is a tree with vertices of degree one or three and $\mu$ is a bijection from the set of leaves of $T$ to $E(G)$. For a subset of edges $X \subseteq E(G)$ let $\delta_{G}(X)$ be the set of all vertices incident to edges in $X$ and $E(G) \backslash X$. For each edge $e$ of $T$, let $T_{1}(e)$ and $T_{2}(e)$ be the sets of leaves in two components of $T \backslash e$. For any edge $e \in E(T)$ we define the middle set as $\operatorname{mid}(e)=\bigcup_{v \in T_{1}(e)} \delta_{G}(\mu(v))$. The width of $\langle T, \mu\rangle$ is the maximum size of a middle set over all edges of $T$, and the branch-width of $G$, bw $(G)$, is the minimum width over all branch decompositions 
of $G$. For a $\mathbb{S}_{0}$-embedded graph $G$, we define a sphere cut decomposition or scdecomposition $\langle T, \mu, \pi\rangle$ as a branch decomposition such that for every edge $e$ of $T$ and the two subgraphs $G_{1}$ and $G_{2}$ induced by the edges in $\mu\left(T_{1}(e)\right)$ and $\mu\left(T_{2}(e)\right)$, there exists a tight noose $O_{e}$ bounding two open discs $\Delta_{1}$ and $\Delta_{2}$ such that $G_{i} \subseteq \Delta_{i} \cup O_{e}, 1 \leq i \leq 2$. Thus $O_{e}$ meets $G$ only in $\operatorname{mid}(e)$ and its length is $|\operatorname{mid}(e)|$. Clockwise traversing of $O_{e}$ in the drawing $G$ defines the cyclic ordering $\pi$ of $\operatorname{mid}(e)$. We always assume that in an sc-decomposition the vertices of every middle set $\operatorname{mid}(e)=V\left(G_{1}\right) \cap V\left(G_{2}\right)$ are enumerated according to $\pi$. The following result follows from the celebrated ratcatcher algorithm due to Seymour and Thomas [13] (the running time of the algorithm was recently improved in [8]; see also [5]).

Proposition 3. Let $G$ be a connected $\mathbb{S}_{0}$-embedded graph without vertices of degree one. There exists an sc-decomposition of $G$ of width $\mathbf{b w}(G)$. Moreover, such a branch decomposition can be constructed in time $O\left(n^{3}\right)$.

\section{$3 \quad$ Hamiltonicity on torus-embedded graphs}

The idea behind solving the HAMILTONIAN CYCLE PROBLEM on $\mathbb{S}_{1}$-embedded graphs is to suitably modify the graph $G$ in such a way that the new graph $G^{\prime}$ is $\mathbb{S}_{0}$-embedded (i.e. planar) and restate the problem to an equivalent problem on $G^{\prime}$ that can be solved by dynamic programming on a sc-decomposition of $G^{\prime}$. As we will see in Section 4, this procedure is extendable to graphs embedded on surfaces of higher genus.

Let $G$ be an $\mathbb{S}_{1}$-embedded graph (i.e. a graph embedded in the torus ). By Proposition 1, it is possible to find in polynomial time a shortest noncontractible (tight) noose $N$ of $G$. Let $G^{\prime}$ be the graph obtained by cutting along $N$ on $G$. By Proposition $2, G^{\prime}$ is $\mathbb{S}_{0}$-embeddible.

Definition 1. A cut of a Hamiltonian cycle $C$ in $G$ along a tight noose $N$ is the set of disjoint paths in $G^{\prime}$ resulting by cutting $G$ along $N$.

Each cut-noose $N_{X}$ and $N_{Y}$ borders an open disk $\Delta_{X}$ and $\Delta_{Y}$, respectively, with $\Delta_{X} \cup \Delta_{Y}=\emptyset$. Let $x_{i} \in N_{X}$ and $y_{i} \in N_{Y}$ be duplicated vertices of the same vertex in $N$.

Definition 2. A set of disjoint paths $\mathbf{P}$ in $G^{\prime}$ is relaxed Hamiltonian if:

(P1) Every path has its endpoints in $N_{X}$ and $N_{Y}$.

(P2) Vertex $x_{i}$ is an endpoint of some path $P$ if and only if $y_{i}$ is an endpoint of a path $P^{\prime} \neq P$.

(P3) For $x_{i}$ and $y_{i}$ : one is an inner vertex of a path if and only if the other is not in any path.

(P4) Every vertex of $G^{\prime} \backslash\left(N_{X} \cup N_{Y}\right)$ is in some path.

A cut of a Hamiltonian cycle in $G$ is a relaxed Hamiltonian set in $G^{\prime}$, but not every relaxed Hamiltonian set in $G^{\prime}$ forms a Hamiltonian cycle in $G$. However, 
given a relaxed Hamiltonian set $\mathbf{P}$ one can check in linear time (by identifying the corresponding vertices of $N_{X}$ and $\left.N_{Y}\right)$ if $\mathbf{P}$ is a cut of Hamiltonian path in $G$. Two sets of disjoint paths $\mathbf{P}=\left(P_{1}, P_{2}, \ldots, P_{k}\right)$ and $\mathbf{P}^{\prime}=\left(P_{1}^{\prime}, P_{2}^{\prime}, \ldots, P_{k}^{\prime}\right)$ are equivalent if for every $i \in\{1,2, \ldots, k\}$, the paths $P_{i}$ and $P_{i}^{\prime}$ have the same endpoints and an inner vertex in one set is also an inner vertex in the other set.

Lemma 1. Let $G^{\prime}$ be a $\mathbb{S}_{0}$-embedded graph obtained from a $\mathbb{S}_{1}$-embedded graph $G$ by cutting along a tight noose $N$. The number of different equivalence classes of relaxed Hamiltonian sets in $G^{\prime}$ is $O\left(\frac{k^{2}}{2} 2^{3 k-2}+2^{3 k}\right)$, where $k$ is the length of $N$.

Proof. In [5] it is argued that the number of non-crossing paths with its endpoints in one noose corresponds to a number of algebraic terms, namely the Catalan numbers. Here we deal with two cut-nooses and our intention is to transform them into one cut-noose. For this, assume two vertices $x_{i} \in N_{X}$ and $y_{j} \in N_{Y}$ being two fixed endpoints of a path $P_{i, j}$ in a relaxed Hamiltonian set $\mathbf{P}$. We look at all possible residual paths in $\mathbf{P} \backslash P_{i, j}$ and we observe that no path crosses $P_{i, j}$ in the $\mathbb{S}_{0}$-embedded graph $G^{\prime}$. So we are able to 'cut' the sphere $\mathbb{S}_{0}$ along $P_{i, j}$ and, that way, create a "tunnel" between $\Delta_{X}$ and $\Delta_{Y}$ unifying them to a single disk $\Delta_{X Y}$. Take the counter-clockwise order of the vertices of $N_{X}$ beginning with $x_{i}$ and concatenate $N_{Y}$ in clockwise order with $y_{j}$ the last vertex. We denote the new cyclic ordering by $\pi_{X Y}$ (see Figure 1 for an example). In $\pi_{X Y}$, let $a, b, c, d$

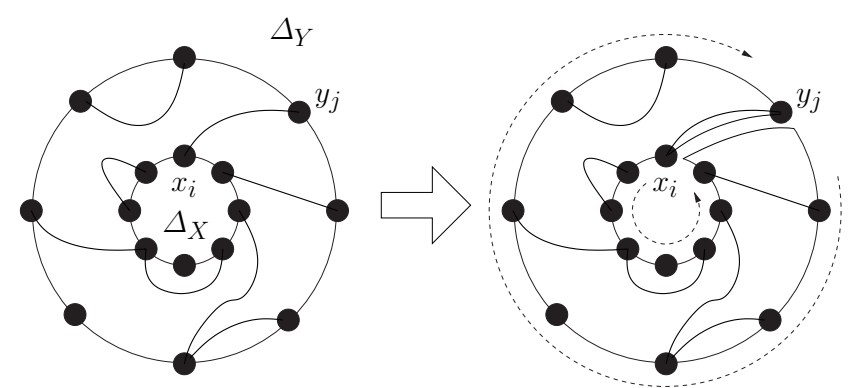

Fig. 1. Cut-nooses. In the left diagram, one equivalence class of relaxed Hamiltonian sets is illustrated. All paths have endpoints in $N_{X}$ and $N_{Y}$. Fix one path with endpoints $x_{i}$ and $y_{i}$. In the right diagram we create a tunnel along this path. The empty disks $\Delta_{X}$ and $\Delta_{Y}$ are united to a single empty disk. Thus, we can order the vertices bordering the disk to $\pi_{X Y}$.

be four vertices where $x_{i}<a<b<c<d<y_{j}$. Notice that if there is a path $P_{a, c}$ between $a$ and $c$, then there is no path between $b$ and $d$ since such a path either crosses $P_{a, c}$ or $P_{i, j}$. This means that we can encode the endpoints of each path with two symbols, one for the beginning and one for the ending of a path. The encoding corresponds to the brackets of an algebraic term. The number of algebraic terms is defined by the Catalan numbers. We say that $\mathbf{P}$ has a Catalan 
structure. With $k=\left|N_{X}\right|=\left|N_{Y}\right|$ and $x_{i}, y_{j} \in P_{i, j}$ fixed, there are $O\left(2^{2 k-2}\right)$ sets of paths having different endpoints and non-crossing $P_{i, j}$. An upper bound for the overall number of sets of paths satisfying (P1) is then $O\left(\frac{k^{2}}{2} 2^{2 k-2}+2^{2 k}\right)$ with the first summand counting all sets of paths for each fixed pair of endpoints $x_{i}, y_{j}$. The second summand counts the number of sets of paths when $N_{X}$ and $N_{Y}$ are not connected by any path. That is, each path has both endpoints in either only $N_{X}$ or only $N_{Y}$. We now count the number of equivalent relaxed Hamiltonian sets $\mathbf{P}$. Apparently, in a feasible solution, if a vertex $x_{h} \in N_{X}$ is an inner vertex of a path, then $y_{h} \in N_{Y}$ does not belong to any path and vice versa. With (P3), there are two more possibilities for the pair of vertices $x_{h}, y_{h}$ to correlate with a path. With $\left|N_{X}\right|=\left|N_{Y}\right|=k$, the overall upper bound of equivalent sets of paths is $O\left(\frac{k^{2}}{2} 2^{3 k-2}+2^{3 k}\right)$.

We call a candidate $\mathbf{C}$ of an equivalence class of relaxed Hamiltonian sets to be a set of paths with vertices only in $N_{X} \cup N_{Y}$ satisfying conditions (P1)(P3). Thus for each candidate we fix a path between $N_{X}$ and $N_{Y}$ and define the ordering $\pi_{X Y}$. By making use of dynamic programming on sc-decompositions we check for each candidate $\mathbf{C}$ if there is a spanning subgraph of the planar graph $G^{\prime}$ isomorphic to a relaxed Hamiltonian set $\mathbf{P}$ such that $\mathbf{P}$ is equivalent to $\mathbf{C}$.

Instead of looking at the HAMILTONIAN CYCLE problem on $G$ we solve the RELAXED HAMILTONiAn SET problem on the $\mathbb{S}_{0}$-embedded graph $G^{\prime}$ obtained from $G$. Given a candidate $\mathbf{C}$ : a set of vertex tuples $\mathbf{T}=\left\{\left(s_{1}, t_{1}\right),\left(s_{2}, t_{2}\right), \ldots,\left(s_{k}, t_{k}\right)\right\}$ with $s_{i}, t_{i} \in N_{X} \cup N_{Y}, i=1, \ldots, k$ and a vertex set $\mathbf{I} \subset N_{X} \cup N_{Y}$. Does there exist a relaxed Hamiltonian set $\mathbf{P}$ such that every $\left(s_{i}, t_{i}\right)$ marks the endpoints of a path and the vertices of $\mathbf{I}$ are inner vertices of some paths? Our algorithm works as follows: first encode the vertices of $N_{X} \cup N_{Y}$ according to $\mathbf{C}$ by making use of the Catalan structure of $\mathbf{C}$ as it follows from the proof of Lemma 1. We may encode the vertices $s_{i}$ as the 'beginning' and $t_{i}$ as the 'ending' of a path of C. Using order $\pi_{X Y}$, we ensure that the beginning is always connected to the next free ending. This allows us to design a dynamic programming algorithm using a small constant number of states. We call the encoding of the vertices of $N_{X} \cup N_{Y}$ base encoding to differ from the encoding of the sets of disjoint paths in the graph. We proceed with dynamic programming over middle sets of a rooted sc-decomposition $\langle T, \mu, \pi\rangle$ in order to check whether $G^{\prime}$ contains a relaxed Hamiltonian set $\mathbf{P}$ equivalent to candidate $\mathbf{C}$. As $T$ is a rooted tree, this defines an orientation of its edges towards its root. Let $e$ be an edge of $T$ and let $O_{e}$ be the corresponding tight noose in $\mathbb{S}_{0}$. Recall that the tight noose $O_{e}$ partitions $\mathbb{S}_{0}$ into two discs which, in turn, induces a partition of the edges of $G$ into two sets. We define as $G_{e}$ the graph induced by the edge set that corresponds to the "lower side" of $e$ it its orientation towards the root. All paths of $\mathbf{P} \cap G_{e}$ start and end in $O_{e}$ and $G_{e} \cap\left(N_{X} \cup N_{Y}\right)$. For each $G_{e}$, we encode the equivalence classes of sets of disjoint paths with endpoints in $O_{e}$. From the leaves to the root for a parent edge and its two children, we update the encodings of the parent middle set with those of the children (for an example of dynamic programming on sc-decompositions, see also [5]). We obtain the algorithm in Figure 2. 


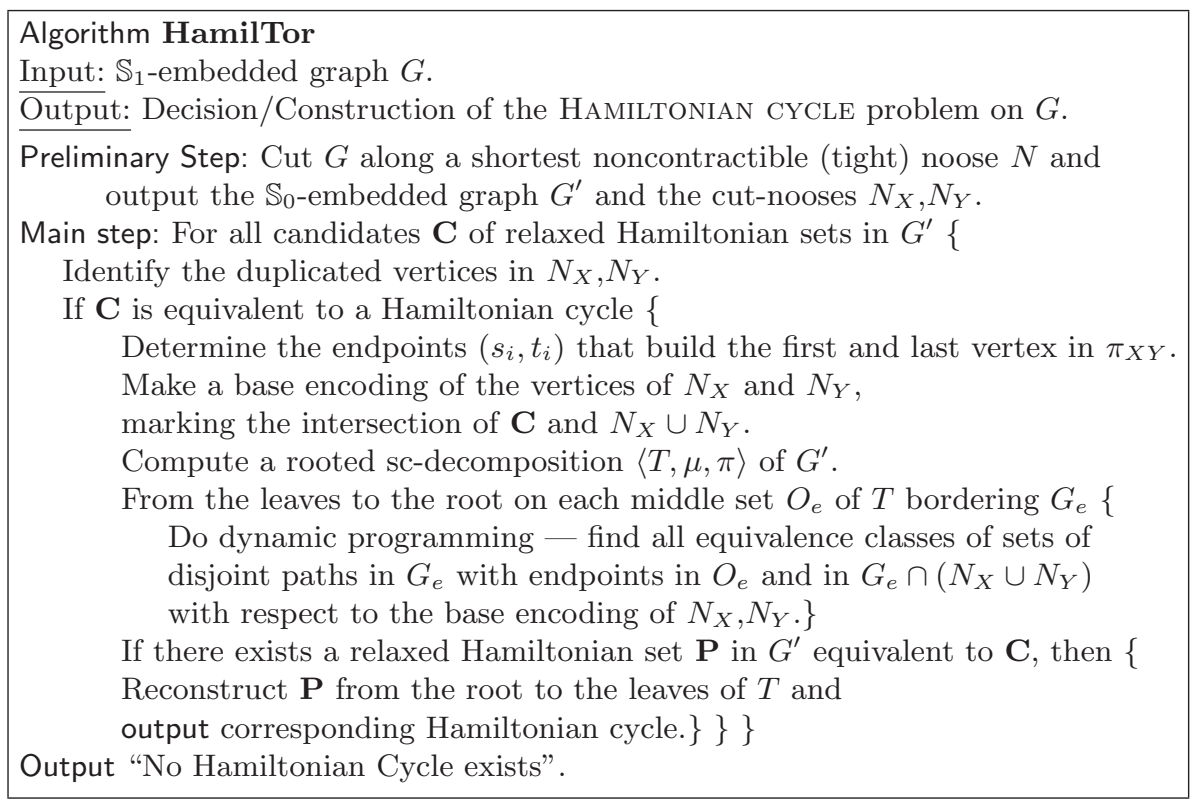

Fig. 2. Algorithm HamilTor.

In the proof of the following lemma we show how to apply the dynamic programming step of HamilTor. The proof is technical, and can be found in the long version of this paper [4]. But we sketch the main idea here: For a dynamic programming step we need the information on how a tight noose $O_{e}$ and $N_{X} \cup N_{Y}$ intersect and which parts of $N_{X} \cup N_{Y}$ are a subset of the subgraph $G_{e}$. Define the vertex set $\mathcal{X}=\left(G_{e} \backslash O_{e}\right) \cap\left(N_{X} \cup N_{Y}\right) . G_{e}$ is bordered by $O_{e}$ and $\mathcal{X} . G_{e}$ is partitioned into several edge-disjoint components that we call partial components. Each partial component is bordered by a noose that is the union of subsets of $O_{e}$ and $\mathcal{X}$. Let us remark that this noose is not necessarily tight. The partial components intersect pairwise only in vertices of $\mathcal{X}$ that we shall define as connectors. In each partial component we encode a collection of paths with endpoints in the bordering noose using Catalan structures. The union of these collections over all partial components must form a collection of paths in $G_{e}$ with endpoints in $O_{e}$ and in $\mathcal{X}$. We ensure that the encoding of the connectors of each two components fit. During the dynamic programming we need to keep track of the base encoding of $\mathcal{X}$. We do so by only encoding the vertices of $O_{e}$ without explicitely memorizing with which vertices of $\mathcal{X}$ they form a path. With several technical tricks we can encode $O_{e}$ such that two paths with an endpoint in $O_{e}$ and the other in $\mathcal{X}$ can be connected to a path of $\mathbf{P}$ only if both endpoints in $\mathcal{X}$ are the endpoints of a common path in $\mathbf{C}$. 
Lemma 2. For a given a sc-decomposition $\langle T, \mu, \pi\rangle$ of $G^{\prime}$ of width $\ell$ and a candidate $\mathbf{C}=(\mathbf{T}, \mathbf{I})$ the running time of the main step of HamilTor on $\mathbf{C}$ is $O\left(2^{5.433 \ell} \cdot\left|V\left(G^{\prime}\right)\right|^{O(1)}\right)$.

To finish the estimation of the running time we need some combinatorial results. The proof of the following two lemmata can be found in [4].

Lemma 3. Let $G$ be a $\mathbb{S}_{1}$-embedded graph on $n$ vertices and $G^{\prime}$ the planar graph after cutting along a noncontractible tight noose. Then $\mathbf{b w}\left(G^{\prime}\right) \leq \sqrt{4.5} \cdot \sqrt{n}+2$.

Lemma 4. Let $G$ be a $\mathbb{S}_{1}$-embedded graph. Then $\operatorname{rep}(G) \leq \sqrt{4.5} \cdot \sqrt{n}+2$.

Putting all together we obtain the following theorem.

Theorem 1. Let $G$ be a graph on $n$ vertices embedded on a torus $\mathbb{S}_{1}$. The HAMILTONIAN CYCLE problem on $G$ can be solved in time $O\left(2^{17.893 \sqrt{n}} \cdot n^{O(1)}\right)$.

Proof. We run the algorithm HamilTor on $G$. The algorithm terminates positively when the dynamic programming is successful for some candidate of an equivalence class of relaxed Hamiltonian sets and this candidate is a cut of a Hamiltonian cycle. By Propositions 1, Step 0 can be performed in polynomial time. Let $k$ be the minimum length of a noncontractible noose $N$, and let $G^{\prime}$ be the graph obtained from $G$ by cutting along $N$. By Lemma 1, the number of all candidates of relaxed Hamiltonian sets in $G^{\prime}$ is $O\left(2^{3 k}\right) \cdot n^{O(1)}$. So the main step of the algorithm is called $O\left(2^{3 k}\right) \cdot n^{O(1)}$ times. By Proposition 3, an optimal branch decomposition of $G^{\prime}$ of width $\ell$ can be constructed in polynomial time. By Lemma 2, dynamic programming takes time $O\left(2^{5.433 \ell}\right) \cdot n^{O(1)}$. Thus the total running time of HamilTor is $O\left(2^{5.433 \ell} \cdot 2^{3 k}\right) \cdot n^{O(1)}$. By Lemma 4, $k \leq \sqrt{4.5} \cdot \sqrt{n}+2$ and by Lemma $3, \ell \leq \sqrt{4.5} \cdot \sqrt{n}+2$, and the theorem follows.

\section{Hamiltonicity on graphs of bounded genus}

Now we extend our algorithm to graphs of higher genus. For this, we use the following kind of planarization: We apply Proposition 2 and cut iteratively along shortest noncontractible nooses until we obtain a planar graph $G^{\prime}$. If at some step $G^{\prime}$ is the disjoint union of two graphs $G_{1}$ and $G_{2}$, we apply Proposition 2 on $G_{1}$ and $G_{2}$ separately. The proof of the following lemma is in [4].

Lemma 5. There exists a polynomial time algorithm that given a $\Sigma$-embedded graph $G$ where $\Sigma \neq \mathbb{S}_{0}$, returns a minimum size noncontractible noose. Moreover, the length of such a noose, $\operatorname{rep}(G)$, is at most $\mathbf{b w}(G) \leq(\sqrt{4.5}+2$. $\sqrt{2 \cdot \operatorname{eg}(\Sigma)}) \sqrt{n}$.

We examine how a shortest noncontractible noose affects the cut-nooses:

Definition 3. Let $\mathcal{K}$ be a family of cycles in $G$. We say that $\mathcal{K}$ satisfies the 3 -path-condition if it has the following property. If $x, y$ are vertices of $G$ and $P_{1}, P_{2}, P_{3}$ are internally disjoint paths joining $x$ and $y$, and if two of the three cycles $C_{i, j}=P_{i} \cup P_{j},(1 \leq i<j \leq 3)$ are not in $\mathcal{K}$, then also the third cycle is not in $\mathcal{K}$. 
Proposition 4. (Mohar and Thomassen [11]) The family of $\Sigma$-noncontractible cycles of a $\Sigma$-embedded graph $G$ satisfies the 3-path-condition.

Proposition 4 is useful to restrict the number of ways not only on how a shortest noncontractible tight noose may intersect a face but as well on how it may intersect the vertices incident to a face. The proof of the following lemma can be found in [4].

Lemma 6. Let $G$ be $\Sigma$-embedded and $F$ a face of $G$ bordered by $V_{1} \subseteq V(G)$. Let $\bar{F}:=V_{1} \cup F$. Let $N_{s}$ be a shortest noncontractible (tight) noose of $G$. Then one of the following holds

1) $N_{s} \cap \bar{F}=\emptyset$.

2.1) $N_{s} \cap F=\emptyset$ and $\left|N_{s} \cap V_{1}\right|=1$.

2.2) $N_{s} \cap F=\emptyset, N_{s} \cap V_{1}=\{x, y\}$, and $x$ and $y$ are both incident to one more face different than $F$ which is intersected by $N_{s}$.

3) $\quad N_{s} \cap F \neq \emptyset$ and $\left|N_{s} \cap V_{1}\right|=2$.

We use Lemma 6 to extend the process of cutting along noncontractible tight nooses such that we obtain a planar graph with a small number of disjoint cutnooses of small lengths. Let $g \leq \operatorname{eg}(\Sigma)$ be the number of iterations needed to cut along shortest noncontractible nooses such that they turn a $\Sigma$-embedded graph $G$ into a planar graph $G^{\prime}$. However, these cut-nooses may not be disjoint. In our dynamic programming approach we need pairwise disjoint cut-nooses. Thus, whenever we cut along a noose, we manipulate the cut-nooses found so far. After $g$ iterations, we obtain the set of cut-nooses $\mathfrak{N}$ that is a set of disjoint cut-nooses bounding empty open disks in the embedding of $G^{\prime}$. Let $L(\mathfrak{N})$ be the length of $\mathfrak{N}$ as the sum over the lengths of all cut-nooses in $\mathfrak{N}$. The proof of the following proposition can be found in [4].

Proposition 5. It is possible to find, in polynomial time, a set of cut-nooses $\mathfrak{N}$ that contains at most $2 g$ disjoint cut-nooses. $L(\mathfrak{N})$ is at most $2 g \operatorname{rep}(G)$.

We extend the definition of relaxed Hamiltonian sets from graphs embedded on a torus to graphs embedded on higher genus, i.e. from two cut-nooses $N_{X}$ and $N_{Y}$ to the set of cut-nooses $\mathfrak{N}$. For each vertex $v$ in the vertex set $V(G)$ of graph $G$ we define the vertex set $D_{v}$ that contains all duplicated vertices $v_{1}, \ldots, v_{f}$ of $v$ in $\mathfrak{N}$ along with $v$. Set $\mathfrak{D}=\bigcup_{v \in V(G)} D_{v}$.

Definition 4. A set of disjoint paths $\mathbf{P}$ in $G^{\prime}$ is relaxed Hamiltonian if:

(P1) Every path has its endpoints in $\mathfrak{N}$.

(P2) If a vertex $v_{i} \in D_{v} \in \mathfrak{D}$ is an endpoint of path $P$, then there is one $v_{j} \in D_{v}$ that is also an endpoint of a path $P^{\prime} \neq P$. All $v_{h} \in D_{v} \backslash\left\{v_{i}, v_{j}\right\}$ do not belong to any path.

(P3) $v_{i} \in D_{v}$ is an inner path vertex if and only if all $v_{h} \in D_{v} \backslash\left\{v_{i}\right\}$ are not in any path.

(P4) Every vertex of the residual part of $G^{\prime}$ is in some path. 
Similar to torus-embedded graphs, we order the vertices of $\mathfrak{N}$ for later encoding in a counterclockwise order $\pi_{\mathbf{L}}$ depending on the fixed paths between the cut-nooses of $\mathfrak{N}$. The proof of the following can be found in [4].

Lemma 7. Let $G^{\prime}$ be the planar graph after cutting along $g \leq \operatorname{eg}(\Sigma)$ tight nooses of $G$ along with its set of disjoint cut-nooses $\mathfrak{N}$. The number of different equivalence classes of relaxed Hamiltonian sets in $G^{\prime}$ is $2^{O(g \cdot(\log g+\operatorname{rep}(G))}$.

Given the order $\pi_{\mathbf{L}}$ of the vertices $\mathfrak{N}$ in the encoding of a candidate $\mathbf{C}$ of a relaxed Hamiltonian set. As in the previous section, we preprocess the graph $G^{\prime}$ and encode the vertices of $\mathfrak{N}$ with the base values. We extend the dynamic porgramming approach by analysing how the tight noose $O_{e}$ can intersect several cut-nooses. The proofs of the next two statements can be found in [4].

Lemma 8. Let $G^{\prime}$ be the planar graph after cutting along $g \leq \operatorname{eg}(\Sigma)$ shortest noncontractible nooses of $G$. For a given sc-decomposition $\langle T, \mu, \pi\rangle$ of $G^{\prime}$ of width $\ell$ and a candidate $\mathbf{C}$ the Relaxed Hamiltonian Set problem on $G^{\prime}$ can be solved in time $2^{O\left(g^{2} \log \ell\right)} \cdot 2^{O(\ell)} \cdot n^{O(1)}$.

Lemma 9. Let $G$ be a $\Sigma$-embedded graph with $n$ vertices and $G^{\prime}$ the planar graph obtained after cutting along $g \leq \mathbf{e g}(\Sigma)$ tight nooses. Then, bw $\left(G^{\prime}\right) \leq$ $\sqrt{4.5} \cdot \sqrt{n}+2 g$.

Lemmata 5, 7, 8 and 9 imply the following:

Theorem 2. Given a $\Sigma$-embedded graph $G$ on $n$ vertices and $g \leq \mathbf{e g}(\Sigma)$. The HAMILTONIAN CYCLE problem on $G$ can be solved in time $n^{O\left(g^{2}\right)} \cdot 2^{O(g \sqrt{g \cdot n})}$.

Our dynamic programming technique can be used to design faster parameterized algorithms as well. For example, the parameterized $p$-CYCLE ON $\Sigma$ EMBEDDED GRAPHS problem asks for a given $\Sigma$-embedded graph $G$, to check for the existence of a cycle of length at least a parameter $p$. First, our technique can be used to find the longest cycle of $G$ with $g \leq \mathbf{e g}(\Sigma)$ in time $n^{O\left(g^{2}\right)} \cdot 2^{O(g \sqrt{g \cdot n})}$. (On torus -embedded graphs this can be done in time $O\left(2^{17.957 \sqrt{n}} n^{3}\right)$.) By combining this running time with bidimensionality arguments from [2] we arrive at a time $2^{O\left(g^{2} \log p\right)} \cdot 2^{O(g \sqrt{g \cdot p})} \cdot n^{O(1)}$ algorithm solving the parameterized $p$-CYCLE ON $\Sigma$-EMBEDDED GRAPHS.

\section{Conclusive Remarks}

In this paper we have introduced a new approach for solving non-local problems on graphs of bounded genus. With some sophisticated modifications, this generic approach can be used to design time $2^{O(\sqrt{n})}$ algorithms for many other problems including $\Sigma$-EMBEDDED GRAPH TSP (TSP with the shortest path metric of a $\Sigma$-embedded graph as the distance metric for TSP), MAX LEAF TREE, and Steiner Tree, among others. Clearly, the ultimate step in this line of research 
is to prove the existence of time $2^{O(\sqrt{n})}$ algorithms for non-local problems on any graph class that is closed under taking of minors. Recently, we were able to complete a proof of such a general result, using results from the Graph Minor series. One of the steps of our proof is strongly based on the results and the ideas of this paper.

\section{References}

1. V. G. DeĬneko, B. Klinz, And G. J. Woeginger, Exact algorithms for the Hamiltonian cycle problem in planar graphs, Operations Research Letters, (2006), p. to appear.

2. E. D. Demaine, F. V. Fomin, M. Hajiaghayi, and D. M. Thilikos, Subexponential parameterized algorithms on graphs of bounded genus and $H$-minor-free graphs, Journal of the ACM, 52 (2005), pp. 866-893.

3. E. D. Demaine And M. HaJiaghayi, Bidimensionality: new connections between FPT algorithms and PTASs, in Proceedings of the 16th Annual ACM-SIAM Symposium on Discrete Algorithms (SODA 2005), New York, 2005, ACM-SIAM, pp. $590-601$.

4. F. Dorn, F. V. Fomin, And D. M. Thilikos, Fast subexponential algorithm for non-local problems on graphs of bounded genus, manuscript, (2006). http://www.ii.uib.no/publikasjoner/texrap/pdf/2006-320.pdf.

5. F. Dorn, E. Penninkx, H. Bodlaender, And F. V. Fomin, Efficient exact algorithms on planar graphs: Exploiting sphere cut branch decompositions, in Proceedings of the 13th Annual European Symposium on Algorithms (ESA 2005), vol. 3669 of LNCS, Springer, Berlin, 2005, pp. 95-106.

6. F. V. Fomin And D. M. Thilikos, New upper bounds on the decomposability of planar graphs, Journal of Graph Theory, 51 (2006), pp. 53-81.

7. J. R. Gilbert, J. P. Hutchinson, and R. E. Tarjan, A separator theorem for graphs of bounded genus, Journal of Algorithms, 5 (1984), pp. 391-407.

8. Q.-P. GU AND H. TAMAKI, Optimal branch-decomposition of planar graphs in $O\left(n^{3}\right)$ time, in Proceedings of the 32nd International Colloquium on Automata, Languages and Programming (ICALP 2005), vol. 3580 of LNCS, Springer, Berlin, 2005, pp. 373-384.

9. R. J. Lipton AND R. E. TARJAn, A separator theorem for planar graphs, SIAM J. Appl. Math., 36 (1979), pp. 177-189.

10. — Applications of a planar separator theorem, SIAM J. Comput., 9 (1980), pp. $615-627$.

11. B. Mohar and C. Thomassen, Graphs on surfaces, Johns Hopkins Studies in the Mathematical Sciences, Johns Hopkins University Press, Baltimore, MD, 2001.

12. N. Robertson And P. D. Seymour, Graph minors. VII. Disjoint paths on a surface, J. Combin. Theory Ser. B, 45 (1988), pp. 212-254.

13. P. D. Seymour And R. Thomas, Call routing and the ratcatcher, Combinatorica, 14 (1994), pp. 217-241.

14. C. Thomassen, Embeddings of graphs with no short noncontractible cycles, J. Combin. Theory Ser. B, 48 (1990), pp. 155-177.

15. G. Woeginger, Exact algorithms for NP-hard problems: A survey, in Combinatorial Optimization - Eureka, you shrink!, vol. 2570 of LNCS, Springer-Verlag, Berlin, 2003, pp. 185-207. 